\title{
Evaluation of Emergency Extracorporeal Shock Wave Lithotripsy for Obstructing Ureteral Stones
}

\author{
Ibrahim F. Ghalayini, Mohammed A. Al-Ghazo, Yousef S. Khader
}

Faculty of Medicine, Jordan University of Science \& Technology, King Abdullah University

Hospital, Irbid, Jordan

\begin{abstract}
Purpose: To assess the efficacy of extracorporeal shockwave lithotripsy (ESWL) for ureteral calculi during acute renal colic.

Materials and Methods: From January 2002 to March 2007, 108 patients were treated by ESWL for obstructing ureteral stones causing acute renal colic. ESWL was performed within 24 hours of the onset of renal colic.

Results: The mean age of the patients was 39.5 years (11-72 years). Male/female ratio was 85/23. Mean stone size was $8.45 \mathrm{~mm}(4-20 \mathrm{~mm})$. They were located in the pelvic $(\mathrm{n}=53)$, iliac $(\mathrm{n}=28)$ or lumbar $(\mathrm{n}=27)$ region. Fragmentation after a single session was complete in 56 patients (52\%), incomplete in 28 (26\%), and absent in 24 (22\%). Patients presenting incomplete fragmentation underwent a second $(n=28)$ or even a third session $(n=11)$. Of the 24 patients in whom ESWL had no impact on the stone, 21 underwent ureteroscopy, and in one case open ureterolithotomy for a patient with a hard $17 \mathrm{~mm}$ stone, while spontaneous passage occurred in two patients with small stones.

Conclusion: Emergency ESWL for obstructing ureteral stones has a satisfactory success rate and very low morbidity. The stone-free rate of retreating ureteral calculi with ESWL decreases significantly after failed initial treatment. Stone size may be the main predictive factor for retreatment. We suggest that no more than 3 treatments should be given for a particular stone due to minimal improvement in the subsequent cumulative treatment success rate.
\end{abstract}

Key words: extracorporeal shockwave lithotripsy; ureteral calculi; emergency; treatment outcome

Int Braz J Urol. 2008; 34: 433-42

\section{INTRODUCTION}

Urinary lithiasis can cause a greater or lesser degree of obstruction of the ureter, depending on the size of the calculus, urothelial edema and the degree of impaction, requiring instrumental treatment, sometimes as an urgent procedure. Optimal treatment for ureteral calculi remains controversial. Treatment options vary and include expectant management, passage of ureteral stent, extracorporeal shockwave lithotripsy (ESWL), ureteroscopy with basket extraction or intracorporeal lithotripsy and open ureterolithotomy. Open surgery is rarely used (1). However, a conservative approach is often complicated by recurrent flank pain, multiple visits to the emergency room (ER), absence from work and an increased risk of serious complications, such as obstruction, infection and silent loss of renal function (2). There is a significant risk of long-term renal impairment if patients have unrelieved obstruction for more than 4 weeks regardless of symptoms and stone size (2). ESWL is the treatment of choice for moderately sized, uncomplicated ureteral 
stones $(3,4)$. It is a simple, robust and safe procedure and is usually recommended for stones resistant to medical treatment in absence of absolute indication of ureteral drainage (5). Interestingly, the role of ESWL as a first line therapy, applied rapidly after the onset of renal colic, has deserved very limited attention. Few studies have suggested that emergency ESWL is an appealing treatment strategy for symptomatic ureteral stones (6-9).

The success rate of ESWL in the treatment of ureteral stones is about $80 \%$ (2). It can be successfully used, without anesthesia, in patients with early recurrence of renal colic (6). Others have used ESWL within 14 days of the onset of acute renal colic but under anesthesia (10) or even during acute renal colic (7) or acute renal failure (11). Moreover, a comparative retrospective analysis has shown that, in emergency situations, ESWL is more effective than nephrostomy or a double J stent and has very low morbidity (12).

We have investigated the efficacy of the lithotripter in the treatment of patients with obstructing ureteral stones during acute renal colic resistant to medical treatment. Also, we compared the success rate of initial shock wave lithotripsy for ureteral calculi with that of subsequent treatments to determine whether more than 1 treatment is justified for any single ureteral stone. Other parameters of treatment outcome were also studied.

\section{MATERIALS AND METHODS}

This study enrolled 108 patients admitted to our department between January 2002 and January 2007 for acute renal colic that proved to be resistant to anti-inflammatory agents or that recurred within 24 hours of such treatment. Admission work-up included: monitoring of vital parameters; temperature; physical examination; blood test for leucocytes, urea, creatinine; urine analysis and culture. All patients should undergo an abdominal X-ray and ultrasound examination. Primary imaging of the patient was performed by helical unenhanced computed tomography of the abdomen, according to current recommendations (13). An intravenous urography (IVU) was only indicated when there was doubt as to the diagnosis. Initial characterization of the stone was based on imaging and included stone size (largest transversal diameter measured by X-ray) and stone location (lumbar, iliac or pelvic ureter).

Patients underwent emergency ESWL using the Dornier lithotripter S (MedTech Europe GmbH, Germany) within $24 \mathrm{~h}$ of admission and the calculi were localized with fluoroscopic guidance. All patients were given sedatives and analgesics and the level of shockwave energy was progressively stepped up until satisfactory stone fragmentation within the limits of patient comfort. Patients for whom the therapeutic modality is contraindicated because of pregnancy, urinary tract infection, coagulation disorders or previous ureteral reimplantation, presence of a perirenal urinoma, temperature $>38 \mathrm{C}$, blood leukocytes $>20,000 / \mathrm{dL}$, solitary kidney, radiolucent stones, or prior history of ureteral stricture or tumor were excluded from the study. Patients with serum creatinine $>1.8 \mathrm{mg} / \mathrm{dL}$, stone located in the renal pelvis or the pyelo-ureteral junction, or if there was any contraindication to ESWL were also excluded.

After defining the indications of treatment, the patients were informed of all the treatment modalities and their probable complications. The need for anesthesia, stent, urethral manipulation, possible complications, need for repeated follow-up especially after ESWL, and the cost factor involved, were explained to the patients.

Baseline medical treatment was started at admission in ER and included IV administration of antispasmodic drug, butylhyoscine $20 \mathrm{mg}$, and intramuscular non-steroid anti-inflammatory drug (NSAID), diclofenac $75 \mathrm{mg}$. Thereafter, diclofenac $75 \mathrm{mg}$ was administered routinely every $12 \mathrm{~h}$.

Lumbar ureteral stones were fragmented with the patient in the supine position, while iliac and pelvic stones in the prone position. At the end of the session, patients completed a visual analog pain scale (0-10). Follow-up over three months comprised evaluation of pain, temperature and fragment elimination, and radiological check-ups (abdominal X-ray and/or ultrasound). Patients in whom ESWL fail to completely disintegrate the stone during a first session underwent repeat sessions. Patients in whom ESWL had no impact on the stone during the first session, as evidenced by abdominal X-ray, were subject to repeated treatment, stent insertion or ureteroscopy. 
Interventional procedures (double $\mathrm{J}$ stent \pm ureteroscopy) were performed within 48-72 hours only in cases of worsening symptoms and impossibility to manage patients medically, appearance of fever or modification in laboratory findings.

Results were compared by the Chi-square test. A 0.05 significance level was used. A mean efficiency quotient (EQ) was calculated according to the formula of Denstedt and co-workers (14): Stone free (\%) X $100 /(100+$ retreatment rate $(\%)+$ rate of auxiliary procedures $(\%))$.

\section{RESULTS}

The mean age of the patients was 39.5 years (11-72 years). Male/female ratio was $85 / 23$. Overall, 21 patients were treated as outpatients and 87 were kept in hospital overnight. All the stones were radiopaque. Their mean size was $8.45 \mathrm{~mm}(4-20 \mathrm{~mm})$.
They were located in the pelvic $(\mathrm{n}=53)$, iliac $(\mathrm{n}=$ $28)$ or lumbar $(n=27)$ region. A total of 108 patients required 163 sessions of lithotripsy with average number of 3710 shock waves at $10-20 \mathrm{kV}$. The mean number of sessions per patient was 1.5 (1-3). The procedure was completed successfully in 106 patients and aborted in 2 patients due to pain. After ESWL treatment, pain resolved in $58 \%$ of patients, persisted in $28 \%$, and required administration of supplementary anti-inflammatory agents or opioids in 14\%. Fragmentation after a single session was complete in 56 patients $(52 \%)$, incomplete in $28(26 \%)$, and absent in $24(22 \%)$. Patient's characteristics at inclusion and in relation to the first session are reported in Table-1.

Patients presenting incomplete fragmentation underwent a second $(n=28)$ or even third session $(n=$ 11). Two patients with remnants after two or three sessions underwent ureteroscopy. One patient developed acute obstructive pyelonephritis proximal to a pelvic stone fragments which was successfully treated by a

Table 1 - Patients characteristics and relation to first ESWL session.

\begin{tabular}{|c|c|c|c|c|c|}
\hline & Total & $\begin{array}{c}\text { Absent } \\
\text { Fragmentation }\end{array}$ & $\begin{array}{c}\text { Incomplete } \\
\text { Fragmentation }\end{array}$ & $\begin{array}{c}\text { Complete } \\
\text { Fragmentation }\end{array}$ & p Value \\
\hline Sex ratio $(M / F)$ & $85 / 23$ & $20 / 4$ & $21 / 7$ & $44 / 12$ & 0.765 \\
\hline Side (L/R) & $56 / 52$ & $13 / 11$ & $14 / 14$ & $29 / 27$ & 0.956 \\
\hline $\begin{array}{l}\text { Age (years) } \\
\text { mean } \\
95 \% \text { CI lower-upper }\end{array}$ & $\begin{array}{c}39.5 \\
37.4,41.6\end{array}$ & $\begin{array}{c}36.8 \\
32.8,40.8\end{array}$ & $\begin{array}{c}38.8 \\
34.0,43.6\end{array}$ & $\begin{array}{c}41.1 \\
38.2,43.9\end{array}$ & 0.256 \\
\hline $\begin{array}{l}\text { Stone location, } \mathrm{n}(\%) \\
\text { Lumbar ureter } \\
\text { Iliac ureter } \\
\text { Pelvic ureter } \\
\text { Total }(\%)\end{array}$ & $\begin{array}{r}27 \\
28 \\
53 \\
108\end{array}$ & $\begin{array}{r}6(22.2) \\
6(21.4) \\
12(22.6) \\
24(22.2)\end{array}$ & $\begin{array}{r}7(25.9) \\
11(39.3) \\
10(18.9) \\
28(25.9)\end{array}$ & $\begin{array}{l}14(51.9) \\
11(39.3) \\
31(58.5) \\
56(51.9)\end{array}$ & 0.372 \\
\hline $\begin{array}{l}\text { Stone size }(\mathrm{mm}) \\
\text { Mean size } \\
\text { Median (range) } \\
4 \text { to }<6 \mathrm{~mm} \\
6 \text { to }<10 \mathrm{~mm} \\
10-20 \mathrm{~mm}\end{array}$ & $\begin{array}{c}8.20 \\
8.0(4,20) \\
19 \\
65 \\
24\end{array}$ & $\begin{array}{c}8.75 \\
8.0(4,17) \\
4 \\
15 \\
6\end{array}$ & $\begin{array}{c}8.93 \\
9.0(5,20) \\
4 \\
19 \\
10\end{array}$ & $\begin{array}{c}7.68 \\
7.0(4,20) \\
11 \\
31 \\
8\end{array}$ & 0.179 \\
\hline $\begin{array}{l}\text { Hospital stay (day) } \\
\text { Mean } \\
\text { Median (range) }\end{array}$ & $\begin{array}{c}2.4 \\
2.0(0,7)\end{array}$ & $\begin{array}{c}4.2 \\
4(0,7)\end{array}$ & $\begin{array}{c}2.4 \\
3.0(0,5)\end{array}$ & $\begin{array}{c}1.6 \\
2.0(0,6)\end{array}$ & $<0.0005$ \\
\hline
\end{tabular}


double $\mathrm{J}$ stent, antibiotics and the fragments passed spontaneously before stent removal. The 24 patients in whom ESWL had no impact on the stone, underwent a second $(n=15)$ or even third session $(n=6)$ without success. Of these, ureteroscopy was performed for 21 cases and open ureterolithotomy for one patient with a hard $17 \mathrm{~mm}$ stone while spontaneous passage occurred in two patients with small stones.

The stone-free success rate for ESWL (fragmentation + elimination) was $31 \%(\mathrm{n}=33)$ on day $2,41 \%(\mathrm{n}=44)$ on day $15,68 \%(\mathrm{n}=73)$ on day 30 , and $77 \%(\mathrm{n}=83)$ on day 90 . The retreatment rate ranged from $28 \%$ to $44 \%$ according to the location of the stone, and from $15.8 \%$ to $66.7 \%$ according to the size of the stone. EQ at 3 months was 49 . Results as a function of stone location and size are given in Table2; both location and size were considered prognostic factors. The mean size of stones that were completely fragmented at a single session $(\mathrm{n}=56)$ was $7.68 \mathrm{~mm}$ $(4-20 \mathrm{~mm})$, of those requiring a second session $(\mathrm{n}=$ 28) was $8.93 \mathrm{~mm}(5-20 \mathrm{~mm})$, and of those resistant to $\operatorname{ESWL}(\mathrm{n}=24)$ was $8.75 \mathrm{~mm}(4-17 \mathrm{~mm})$. There were no major complications, although eleven patients mentioned macroscopic hematuria afterwards, none requiring specific treatment which is an expected side-effect to treatment.

Group analysis were performed by combining stone location (lumbar vs. iliac or pelvic) and size (largest diameter $<6,6$ to $<10 \mathrm{~mm}$ or $10-20 \mathrm{~mm}$ ). The amplitude of the benefit, however, was more stringent for stones located proximally and with a size $>5 \mathrm{~mm}$.
Median and average hospital stay were 2.0 and 2.4 days (95\% lower and upper confidence interval: 2.1-2.8 days). This effect largely depended on the rate of fragmentation after the first session as well as the size and location of the stone.

We were able to analyze stones from 32 patients in the study. The majority of the patients had calcium oxalate stones $(n=20)$ while the remainder had mixed calcium oxalate and phosphate $(\mathrm{n}=6)$, struvite $(n=5)$, and cystine stones $(n=1)$.

\section{COMMENTS}

In the last 20 years, the development and constant improvement of minimally invasive techniques such as ureteroscopy with in situ lithotripsy or laser fragmentation and ESWL has prompted urologists toward a more aggressive attitude. Although observation is still recommended for stones measuring less than 4 $\mathrm{mm}$ in diameter, most international guidelines today recommend active removal of all stones exceeding 5-7 $\mathrm{mm}$, when proven that they have resisted medical therapy (9). The spontaneous rate of elimination of the stones depends on the stone size and position in the ureter (2). In a recent prospective study using unenhanced helical CT, Coll et al. have demonstrated that the spontaneous passage rate for stones ranged from $87 \%$ to $25 \%$ according to the size of stones (1 $\mathrm{mm}$ in diameter to more than $9 \mathrm{~mm}$ ) (15). In the same series, spontaneous passage rate was also dependent on stone location ( $48 \%$ for stones in the proximal

Table 2 - Results of ESWL at 3 months as a function of stone location and size.

\begin{tabular}{lrcccc}
\hline & N & Mean Size & Success Rate (\%) & Retreatment Rate (\%) & $\begin{array}{c}\text { Hospital Stay } \\
\text { Mean (SD) }\end{array}$ \\
\hline Size & & & & & \\
$\quad$ Total & 108 & 8.2 & $82(76.9)$ & $38(35.2)$ & $2.4(1.8)$ \\
4 to $<6 \mathrm{~mm}$ & 19 & 4.7 & $13(84.2)$ & $3(15.8)$ & $1.5(1.8)$ \\
$\quad$ to $<10 \mathrm{~mm}$ & 63 & 7.6 & $65(77)$ & $19(29.2)$ & $2.2(1.7)$ \\
$\quad 10-20 \mathrm{~mm}$ & 24 & 12.5 & $17(70.8)$ & $16(66.7)$ & \\
Location & & & & & \\
$\quad$ Lumbar & 27 & 9.6 & $22(81.5 \%)$ & $12(44.4)$ & $3.1(1.7)$ \\
Iliac & 28 & 7.7 & $21(75.0 \%)$ & $11(39.3)$ & $2.1(1.7)$ \\
$\quad$ Pelvic & 53 & 7.8 & $40(75.5 \%)$ & $15(28.3)$ & $(1.8)$ \\
\hline
\end{tabular}


ureter, $60 \%$ for mid ureteral stones, $75 \%$ for distal stones, and $79 \%$ for ureterovesical junction stones). In addition to size and location, there are also other interfering factors such as obesity, level of renal obstruction and type of medical therapy (16). In our study, most of the stones and fragments that passed spontaneously were $7 \mathrm{~mm}$ or less and located in the lower ureter. Active removal is also strongly indicated in patient with persistent pain despite adequate medical treatment, acute obstruction with impaired renal function or solitary functional kidney, urinary tract infection, risk or suspicion of urosepsis $(2,17)$. In cases where removal of ureteral stone is warranted, the main debate centers currently around the choice of ESWL or endoscopic management combined with laser or mechanic fragmentation $(4,18,19)$.

Traditionally, the imaging study used for evaluating patients presenting with ureteral colic believed secondary to an acute episode was IVU. Although the examination was often diagnostic, limitations included inability to obtain proper bowel preparation to aid in imaging because of the acute nature of the study, risk of allergy to contrast agents, potential nephrotoxicity, need to assess renal function before contrast injection, inability of conventional radiography to visualize some stones (e.g., uric acid), and the time-consuming nature of the study. Though renal ultrasonography is sometimes useful in detecting the presence of hydronephrosis secondary to an obstructing ureteral stone, the evaluation is very operator dependent. Furthermore, the study is unable to accurately measure the size of the stone and locate ureteral stones in many instances. Computer tomography $(\mathrm{CT})$ scan is able to address many of these issues and, with the introduction of spiral CT, nonenhanced studies are rapidly becoming the standard means of evaluating patients presenting to emergency departments with acute flank pain (13).

In institutions equipped with ESWL the question arises whether applying ESWL shortly after the onset of renal colic could help resolving this issue. Interestingly enough, although ESWL is widely considered as one of the treatments of choice of ureteral stones, its use as an immediate therapeutic tool in an ER setting has not yet deserved much attention. To our knowledge, only reports by Gonzalez Enguita et al. (8), Doublet et al. (6), Tligui et al. (7), and Tombal et al. (9) have addressed its potential interest. Tligui et al. reported in 2003 their experience of 200 patients suffering from acute renal colic and treated with emergency ESWL (EDAP LT-02) within $24 \mathrm{~h}$. Stone-free rate ranged from $79 \%$ to $83 \%$ according to the location of the stone, and from $75 \%$ to $86 \%$ according to the size of the stone. Two or three ESWL sessions were required in 79 patients. The 36 patients, in whom ESWL failed, underwent ureteroscopy $(n=23)$ or lithotripsy with a Dornier ${ }^{\mathbb{B}}$ machine $(\mathrm{n}=13)$. Based on this observation, they advocated a more widespread use of the technique based on a high stone free rates after three months and a low morbidity. These are consistent with our findings. The study however was not randomized. We could not do a randomization of our patients in order to collect a representative number of patients to undergo statistical workup.

Tombal et al. in 2005 reported the results of the first randomized trial addressing the role of emergency ESWL in 100 patients requiring hospitalization for the management of renal colic (9). These authors have prospectively compared standard medical treatment with NSAID and antispasmodic to medical treatment plus emergency ESWL, performed without analgesia on a Siemens Lithostar lithotripter (Siemens Medical Systems, AG, Munich, Germany) within 6 $h$. following admission to the ER. On average, this study showed that ESWL increased the proportion of patients stone-free (SF) after 48 hours (SF-48) by $13 \%$ while it increased the median duration of hospitalization by one day. Emergency ESWL increased both SF-48 and proportion of patients discharged from the hospital at 72 hours by respectively $40 \%$ and $25 \%$ when the stone was located proximally and $>5 \mathrm{~mm}$, and they advocated that it should be strongly recommended in these cases. In contrast, when the stone is located distally from the crossing of the iliac artery, ESWL only slightly increased stone free rate by $5 \%$ while decreasing the proportion of patients released from hospitalization at $48 \mathrm{~h}$ and $72 \mathrm{~h}$. Their study demonstrated that emergency ESWL is a valuable therapeutic option to improve elimination of ureteral stones and shorten duration of hospital stay, when proven that the stone is located proximally to the iliac vessels.

A better outcome of ESWL has been reported for kidney stones compared to ureter stones, while 
others could not demonstrate such differences $(20,21)$. Pace et al. investigated a large number of ESWL cases and demonstrated a superior success rate for upper and mid ureter stones compared to distal calculi (22). The AUA meta-analysis revealed best stone clearance for small stones $<10 \mathrm{~mm}$, with $74 \%$ compared to $46 \%$ for stones between 11-20 mm (2). For complete stone disintegration, many patients have to undergo 2 or more shockwave sessions (2). There is reported no consensus on the number of shock wave lithotripsy treatments for ureteral calculi that should be administered for a single stone before alternate modalities are used. Pace et al. (22) have reported a low success rate of repeat shock wave lithotripsy for ureteral stones after failed initial treatment. Kim et al. suggested that no more than 3 treatments should be given for a particular stone due to minimal improvement in the subsequent cumulative treatment success rate (23). We compared the success rate of initial shock wave lithotripsy for ureteral calculi with that of subsequent treatments to determine whether more than 1 treatment is justified for any single ureteral stone. In this respect our results are in agreement with the other reported series as none of our patients responded to repeat sessions after failure of the initial treatment. In a series of 1588 patients they had treated 1593 ureteral calculi with the Dornier MFL 5000 lithotripter (Dornier Medical Systems Inc., Kennesaw, GA) over a period from January 1994 to September 1999 (22). The stone free rate after initial treatment was 68\% (1086 of 1593 stones), which decreased to $46 \%$ for first re-treatment and $31 \%$ for second re-treatment. Overall the success rate increased to $77 \%$ after 3 treatments compared with $76 \%$ after two treatments. Upper and mid ureter stone free rates were significantly higher than those in the lower ureter after initial treatment. Success rate was also greater for smaller stones $(10 \mathrm{~mm}$ or less versus 11 to $20 \mathrm{~mm}$ was $74 \%$ versus $43 \%$ ( $<<0: 001)$. In our series, those patients with incomplete fragmentation after the initial treatment were not offered more than 3 sessions of ESWL and all of them were stone free by 3 months. We found that the stone free rate was higher for smaller stones $(9 \mathrm{~mm}$ or less versus 10 to $20 \mathrm{~mm}$ was $78.6 \%$ versus $70.8 \%, \mathrm{p}=0.428$ ). Although the difference was not significant, hospital stay was significantly higher for the large stones (mean; 3.7 vs. $2.1, \mathrm{p}<0.0005)$. It was also significantly higher for the lumber ureter $(\mathrm{p}=0.016)$ as the stone size increased in the proximal ureter. Upper stone free rate $(81.5 \%)$ was higher than those in the mid and lower ureter $(75 \%$ and $75.5 \%$, respectively $(\mathrm{p}=0.804))$ after initial treatment with higher retreatment rate. The rate of retreatment depends on the stone size and position in the ureter. It increased for upper ureteric stones $(37 \%)$ compared to the mid and lower ureteric stones (28.6\% and $24.5 \%$, respectively). This may be explained by the higher mean stone size $(9.6 \mathrm{~mm})$ for upper ureter compared to the mid and lower ureter (7.7 and $7.8 \mathrm{~mm}$, respectively). Also, we found difficulty in localization for some cases with mid ureteric stones as overlapped by the iliac bone. Retreatment for a ureteric stone appeared to increase the stone free rate of initial treatment from $58 \%$ to $77 \%$. It may be that stone size is the main predictor factor for the retreatment rate.

With the widespread use of ESWL, fewer stones are being analyzed because of difficulties in collecting stone samples. We were able to analyze stones from 32 patients and calcium oxalate stones were the most common type.

More commonly, hospitalization is required to manage intractable pain resistant to oral or intrarectal therapy. While the main goal of therapy should then still be oriented toward fast pain relief and safe stone removal, it is also critical to achieve rapid discharge from the hospital. In our series the majority of the patients had treatments as an inpatient procedure (81\%) mainly for 'social' reasons, i.e. ,difficulty in transport, lack of follow-up, health care facility and less commonly for complications. Overall although, there is still considerable scope for improving the process of supplying emergency interventional care and reducing inpatient stay.

Ureteral pre-stenting is only necessary for patients with persistent pain, fever or renal insufficiency due to obstruction. Some authors reported a decreased stone free rate after introduction of an indwelling stent, most probably due to problems in stone detection and interference with the shock waves $(4,22)$. Especially with older lithotripters, focusing on ureter stones was difficult. For this reason pre-stenting was not part of our treatment. If practical, in situ shockwave lithotripsy in acute obstructive ureteric lithiasis seems to be advantageous compared to later 
shockwave application in the non-obstructive phase ${ }^{28}$. Arrabal-Martın et al. recently demonstrated, that in situ ESWL for both obstructive and non-obstructive lumbar ureter stones reached $95.5 \%$ and $93.15 \%$ stone free rate respectively (4).

As kidney stones were thought to show a better response to ESWL, push-back manipulation into the kidney was recommended for proximal ureter stones. We do not recommend this as with improved lithotripsy and stone detection technology, this procedure is now considered being out-dated. Some investigators (21) have reported a better outcome of ESWL after stone manipulation, while others (20) have not found a statistical difference. However it can prove difficult to manipulate an impacted stone, and the possibility of post-treatment obstruction by a large fragment in an edematous ureter remains. This risk can be minimized by stent placement at the time of stone manipulation. Advances in ureteroscopic technology with the introduction of small caliber semi-rigid and flexible ureteroscopes combined with the introduction of the holmium YAG laser have improved stone free rates following ureteroscopy while decreasing the risk of complications $(24,25)$.

Success rates for shock wave lithotripsy may differ according to the lithotripter used. Average stonefree rate for cumulative shock wave lithotripsy series reported in the literature using an HM3 lithotripter is slightly but consistently higher than that achieved with many second and third generation lithotripters and may influence the choice of treatment (26). It is important to stress that the results with shock wave lithotripsy are truly machine specific and cannot be translated to use with other lithotripters (19). The Dornier Lithotripter S that we use proved in different series to be very effective in the treatment of renal and ureteral calculi (18).

In conclusion, rapidly performed ESWL is a valuable therapeutic option to improve elimination of ureteral stones. We agree with the other authors that it could be more widespread in acute renal colic. It presents medical advantages, i.e. no need for prolonged anti-inflammatory treatment, and also possible economic advantages, i.e. no need for anesthesia and routine hospitalization with fewer absences from work. It requires appropriate lithotripter facilities for emergency use and a follow-up period of up to three months. Ultimately, the chosen treatment option (medical treatment, ESWL, or ureteroscopy) is a matter of a joint decision between the physician and the informed patient.

\section{CONFLICT OF INTEREST}

None declared.

\section{REFERENCES}

1. Assimos DG, Boyce WH, Harrison LH, McCullough DL, Kroovand RL, Sweat KR: The role of open stone surgery since extracorporeal shock wave lithotripsy. J Urol. 1989; 142: 263-7.

2. Segura JW, Preminger GM, Assimos DG, Dretler SP, Kahn RI, Lingeman JE, et al.: Ureteral Stones Clinical Guidelines Panel summary report on the management of ureteral calculi. The American Urological Association. J Urol. 1997; 158: 1915-21.

3. Tiselius HG, Ackermann D, Alken P, Buck C, Conort P, Gallucci M, et al.: Guidelines on urolithiasis. Eur Urol. 2001; 40: 362-71.

4. Arrabal-Martín M, Pareja-Vilches M, Gutiérrez-Tejero F, Miján-Ortiz JL, Palao-Yago F, Zuluaga-Gómez A: Therapeutic options in lithiasis of the lumbar ureter. Eur Urol. 2003; 43: 556-63.

5. Tombolini P, Ruoppolo M, Bellorofonte C, Zaatar C, Follini M: Lithotripsy in the treatment of urinary lithiasis. J Nephrol. 2000; 13 (Suppl 3) : S71-82.

6. Doublet JD, Tchala K, Tligui M, Ciofu C, Gattegno B, Thibault P: In situ extracorporeal shock wave lithotripsy for acute renal colic due to obstructing ureteral stones. Scand J Urol Nephrol. 1997; 31: 137-9.

7. Tligui M, El Khadime MR, Tchala K, Haab F, Traxer $\mathrm{O}$, Gattegno B, et al.: Emergency extracorporeal shock wave lithotripsy (ESWL) for obstructing ureteral stones. Eur Urol. 2003; 43: 552-5.

8. González Enguita C, Cabrera Pérez J, Calahorra Fernández FJ, García Cardoso J, Vela Navarrete R. Efficient, immediate or emergency ESWL: an attractive strategic alternative to be considered in the treatment of renal colic! Actas Urol Esp. 2000; 24: 721-7.

9. Tombal B, Mawlawi H, Feyaerts A, Wese FX, Opsomer $\mathrm{R}$, Van Cangh PJ: Prospective randomized evaluation of emergency extracorporeal shock wave lithotripsy (ESWL) on the short-time outcome of symptomatic 
ureteral stones. Eur Urol. 2005; 47: 855-9. Erratum in: Eur Urol. 2005; 48: 876.

10. Cass AS: In situ extracorporeal shock wave lithotripsy for obstructing ureteral stones with acute renal colic. J Urol. 1992; 148: 1786-7.

11. Numa H, Yoshida K, Kageyama Y, Hoshino Y. In situ extracorporeal shock wave lithotripsy for ureteral stones causing acute renal failure. Hinyokika Kiyo. 1994; 40: 291-4.

12. Joshi HB, Obadeyi OO, Rao PN: A comparative analysis of nephrostomy, JJ stent and urgent in situ extracorporeal shock wave lithotripsy for obstructing ureteric stones. BJU Int. 1999; 84: 264-9.

13. Wang LJ, Ng CJ, Chen JC, Chiu TF, Wong YC: Diagnosis of acute flank pain caused by ureteral stones: value of combined direct and indirect signs on IVU and unenhanced helical CT. Eur Radiol. 2004; 14 : 1634-40.

14. McDougall EM, Denstedt JD, Brown RD, Clayman RV, Preminger GM, McClennan BL: Comparison of extracorporeal shock wave lithotripsy and percutaneous nephrolithotomy for the treatment of renal calculi in lower pole calices. J Endourol. 1989; 3: 265-71.

15. Coll DM, Varanelli MJ, Smith RC: Relationship of spontaneous passage of ureteral calculi to stone size and location as revealed by unenhanced helical CT. AJR Am J Roentgenol. 2002; 178: 101-3.

16. Deliveliotis C, Chrisofos M, Albanis S, Serafetinides E, Varkarakis J, Protogerou V: Management and follow-up of impacted ureteral stones. Urol Int. 2003; 70 : 269-72.

17. Anagnostou T, Tolley D: Management of ureteric stones. Eur Urol. 2004; 45: 714-21.
18. Ghalayini IF, Al-Ghazo MA, Khader YS: Extracorporeal shockwave lithotripsy versus ureteroscopy for distal ureteric calculi: efficacy and patient satisfaction. Int Braz J Urol. 2006; 32: 656-64; discussion 664-7.

19. Hochreiter WW, Danuser H, Perrig M, Studer UE: Extracorporeal shock wave lithotripsy for distal ureteral calculi: what a powerful machine can achieve. J Urol. 2003; 169: 878-80.

20. Rassweiler J, Lutz K, Gumpinger R, Eisenberger F: Efficacy of in situ extracorporeal shock wave lithotripsy for upper ureteral calculi. Eur Urol. 1986; 12: 377-86.

21. Graff J, Pastor J, Funke PJ, Mach P, Senge T: Extracorporeal shock wave lithotripsy for ureteral stones: a retrospective analysis of 417 cases. J Urol. 1988; 139: 513-6.

22. Pace KT, Weir MJ, Tariq N, Honey RJ: Low success rate of repeat shock wave lithotripsy for ureteral stones after failed initial treatment. J Urol. 2000; 164: 1905-7.

23. Kim HH, Lee JH, Park MS, Lee SE, Kim SW: In situ extracorporeal shockwave lithotripsy for ureteral calculi: investigation of factors influencing stone fragmentation and appropriate number of sessions for changing treatment modality. J Endourol. 1996; 10: 501-5.

24. Devarajan R, Ashraf M, Beck RO, Lemberger RJ, Taylor MC: Holmium: YAG lasertripsy for ureteric calculi: an experience of 300 procedures. Br J Urol. 1998; 82: 342-7.

25. Scarpa RM, De Lisa A, Porru D, Usai E: Holmium:YAG laser ureterolithotripsy. Eur Urol. 1999; 35: 233-8.

26. Gettman MT, Segura JW: Management of ureteric stones: issues and controversies. BJU Int. 2005; 95 (Suppl 2): 85-93.

\author{
Correspondence address: \\ Dr. Ibrahim Fathi Ghalayini \\ Associate Professor of Urology \\ P.O. Box 940165 \\ Amman, 11194, Jordan \\ Fax: + 0096265687422 \\ E-mail: ibrahimg@just.edu.jo
}




\section{EDITORIAL COMMENT}

We read with interest this original article about the role of shock wave lithotripsy (ESWL) as an emergency treatment of ureteral lithiasis. Unfortunately, while the debate between ESWL and ureteroscopy for such stones is still going on, the importance of ESWL as an emergency approach to face this problem has been merely evaluated (1-3). Wherever lithotripters are available, ESWL may represent the non invasive way to perform an active stone removal, attempting to resolve this common and potentially severe emergency. Its safety leads to an ease of use in most of the cases, excluding those with absolute contraindications (pregnancy, uncorrected bleeding disorders) or complicated features (i.e. ureteral stones associated with urosepsis and /or severe renal function impairment).

One of the crucial points of emergency ESWL trials is the choice of a proper end point: fragmentation and expulsion are achieved gradually after ESWL, and those phases, above all the expulsive one, can last a considerable and variable period of time, depending on stone size, location and ureteral edema. Due to this last consideration, ESWL of ureteral stone is highly recommended within a short period from the onset of acute renal colic. In fact, in uncomplicated cases, like the ones reported in this series, a satisfying stone free rate is rapidly achieved after the treatment. Moreover, one of the main outcomes of this study is that pain was rapidly and definitively controlled after ESWL in $58 \%$ of the cases, thus allowing a watchful waiting approach of spontaneous passage of the fragments. Pain relief may enable a faster discharge, and even if only 21 were treated as outpatients, Authors invoked an improve in the delivery process that can be easily achieved. Furthermore, hospitalization, its length and relationship with stone characteristics were properly analyzed in this manuscript.

Since renal colic due to stone disease is a widespread problem, we have previously assessed the role of ESWL as an emergency treatment of ureteral stones associated with mild renal function impairment. Our outcomes focused on the ability of a single session ESWL to decrease rapidly creatinine serum levels, and a normalization of such parameter was evident in $85 \%$ of the patients 24 hours after the treatment. A complete stone free condition was then reached gradually ( $67.5 \%$ at 72 hours), and 7 out of 40 patients underwent a successful second session ESWL. Characteristics were properly analyzed in this manuscript.

Since renal colic due to stone disease is a widespread problem, we have previously assessed the role of ESWL as an emergency treatment of ureteral stones associated with mild renal function impairment (4). Our outcomes focused on the ability of a single session ESWL to decrease rapidly creatinine serum levels, and a normalization of such parameter was evident in $85 \%$ of the patients 24 hours after the treatment. A complete stone free condition was then reached gradually ( $67.5 \%$ at 72 hours), and 7 out of 40 patients underwent a successful second session ESWL (4).

Except for this last consideration, our findings are consistent with those reported by the Authors, as ESWL turns out to be effective even as an emergency procedure, potentially reducing the need for an endoscopic management.

Few minor concerns remain, i.e. the tricky focusing of ureteral stones overlapping iliac bone and the role of ureteral stenting, that still represents a matter of debate. Furthermore, we believe that the definite role of repeated sessions have yet to be defined, and greater series in a prospective setting have to assess the value of ESWL multiple treatments.

\section{REFERENCES}

1. Kravchick S, Bunkin I, Stepnov E, Peled R, Agulansky L, Cytron S: Emergency extracorporeal shockwave lithotripsy for acute renal colic caused by upper urinary-tract stones. J Endourol. 2005; 19: 1-4.

2. Doublet JD, Tchala K, Tligui M, Ciofu C, Gattegno B, Thibault P: In situ extracorporeal shock wave lithotripsy for acute renal colic due to obstructing ureteral stones. Scand J Urol Nephrol. 1997; 31: 137-9.

3. Tligui M, El Khadime MR, Tchala K, Haab F, Traxer O, Gattegno B, et al.: Emergency extracorporeal shock wave lithotripsy (ESWL) for obstructing ureteral stones. Eur Urol. 2003; 43: 552-5. 
4. Sighinolfi MC, Micali S, DE Stefani S, Grande M, Saredi G, Mofferdin A, et al.: Noninvasive management of obstructing ureteral stones using electromag- netic extracorporeal shock wave lithotripsy. Surg Endosc. 2008 (In press).

Dr. M. C. Sighinolfi, Dr. S. Micali Dr. S. De Stefani \& Dr. G. Bianchi Department of Urology University of Modena and Reggio Modena, Emilia, Italy E-mail:sighinolfic@yahoo.com 